\title{
Perspective
}

PERSPECTIVE Actualité en histoire de l'art

4 | 2006

La monographie d'artiste

\section{Kurt Schwitters, entre rigueur scientifique et souffle poétique}

\section{Isabelle Ewig}

\section{(2) OpenEdition}

1 Journals

\section{Édition électronique}

URL : http://journals.openedition.org/perspective/4229

DOI : 10.4000/perspective.4229

ISSN : 2269-7721

Éditeur

Institut national d'histoire de l'art

\section{Édition imprimée}

Date de publication : 31 décembre 2006

Pagination : 642-645

ISSN : 1777-7852

\section{Référence électronique}

Isabelle Ewig, « Kurt Schwitters, entre rigueur scientifique et souffle poétique », Perspective [En ligne],

4 | 2006, mis en ligne le 31 mars 2018, consulté le 01 octobre 2020. URL : http://

journals.openedition.org/perspective/4229; DOI : https://doi.org/10.4000/perspective.4229

Ce document a été généré automatiquement le 1 octobre 2020. 


\title{
Kurt Schwitters, entre rigueur scientifique et souffle poétique
}

\author{
Isabelle Ewig
}

1 Dans l'abondante littérature sur Kurt Schwitters, l'approche monographique a souvent été privilégiée. Cela tient sans doute à l'œuvre et à la vie de l'artiste, et à la façon dont l'un et l'autre s'articulent. Rappelons en effet que l'activité de Schwitters couvre plus de quatre décennies (de ses premiers essais, en 1905, à sa mort, en 1948) et qu'elle ne connaît pas de passage à vide, l'œuvre tardif n'ayant rien à envier à la période dite «classique », celle inaugurée par la création, en 1919, de son propre mouvement: MERZ. Un nom de baptême issu d'un tableau, où se lit l'intitulé Merz, fragment découpé dans une annonce pour la Privat- und Kommerzbank puis collé sur la toile ; par la suite, ce vocable devait qualifier toutes les formes de la création de Schwitters, collages, assemblages, sculptures, architecture, poésie, typographie, jusqu'à englober sa propre personne: «À présent, écrivait-il en 1927, je m'appelle moi-même Merz ». L'identification entre l'artiste et son œuvre (pas d'autre adhérent à Merz que son créateur) invite à mettre au jour la singularité du parcours et du destin de Schwitters; on plonge alors au cœur du bouillonnement intellectuel et artistique de l'Europe d'après le premier conflit mondial, on traverse l'histoire sombre de l'Allemagne, on le suit dans l'expérience douloureuse de l'exil, en Norvège d'abord, en Angleterre ensuite.

2 Parmi les ouvrages récemment publiés sur Schwitters, certains permettent de préciser ces aspects, tout en offrant - ou non - de nouvelles interprétations. Ainsi la publication $\mathrm{du}$ catalogue raisonné ${ }^{1}$ confirme-t-elle, si besoin était, l'originalité de l'artiste, en donnant à la période tardive et à l'œuvre figuratif sa juste mesure. Complétant le catalogue raisonné, dont l'objet d'étude est, par définition, exclusivement la production de l'artiste, deux catalogues d'exposition rendent compte de la place de premier plan que Schwitters occupe dans l'art de son temps, l'un en retraçant ses relations avec « ses amis » (celui du Sprengel Museum à Hanovre) ${ }^{2}$, l'autre en analysant et comparant sa démarche artistique à celle de Jean Arp (celui du Kunstmuseum à Bâle) ${ }^{3}$. Enfin, la période des exils est relatée comme jamais auparavant par Patrick Beurard-Valdoye . $^{4}$ Aussi éloignés soient-ils dans leur forme, le catalogue raisonné et la prose poétique de 
P. Beurard-Valdoye se rejoignent dans la rigueur scientifique et s'inscrivent tous deux dans les pas de Schwitters, qui avait un avis bien tranché sur la façon dont il convenait de gérer et de commenter son œuvre.

3 Schwitters est à mille lieues de la conception de l'art des dadaïstes, avec lesquels il a pourtant entretenu de nombreux liens. Ceux-ci avaient une dent contre l'art - cela s'est traduit entre autres par l'adoption d'une terminologie en rupture avec la tradition: " photomonteur ", «foire", " productions » plutôt que "peintre ", « exposition », «œuvres d'art». Pourtant, force est de constater que Dada a créé quelques-uns des chef-d'œuvres du xxe siècle. Cette contradiction, aujourd'hui parfaitement assumée par les historiens de l'art et les commissaires d'exposition, ne se retrouve pas chez Schwitters. En effet, bien qu'utilisant colle et clous, il revendique le statut de " peintre » et, dans la foulée, adopte la terminologie en usage, qualifiant ses collages de dessins (Merzzeichnung) et ses assemblages de tableaux (Merzbild). Il prend soin de les assortir pour les premiers d'un passe-partout, pour les seconds d'un cadre, de les signer, de les dater et de les intituler. Il pense aussi à les documenter. Ainsi, en 1920, préparant une exposition au Sturm, il suggère à Herwarth Walden de "faire entrer dans le catalogue non seulement les œuvres exposées mais aussi l'ensemble des œuvres vendues, données ou accessibles au public par reproduction à ce jour $»^{5}$, en indiquant leur lieu de conservation ou leur support de diffusion. Au-delà de la motivation commerciale qu'il avoue, il a en tête de garder la trace de ses œuvres. Leur pérennité lui importe. D'ailleurs aux heures les plus sombres de l'Histoire, il a tenté de les préserver, emmurant son Merzbau (mais un bombardement en eut raison), transportant et entreposant collages et assemblages à Lysaker, près d'Oslo, où il s'était réfugié. Les caisses, laissées sur place au moment de sa fuite vers l'Angleterre en 1940, ont été ouvertes à ce même endroit environ quinze ans plus tard, par Werner Schmalenbach et le fils de l'artiste, Ernst Schwitters ${ }^{6}$. Les efforts de l'artiste n'avaient donc pas été totalement vains. Aujourd'hui, d'autres que lui assurent la pérennité de son œuvre, notamment à travers la publication du catalogue raisonné : édité par Hatje Cantz en trois volumes parus successivement en 2000, 2003 et 2006, il résulte d'un travail de titan mené depuis une dizaine d'années par les Dr. Karin Orchard et Isabel Schulz au sein du Sprengel Museum de Hanovre. Sis dans la ville natale de Schwitters, ce musée est en possession de 117 de ses œuvres et en abrite 1010, déposées par la Kurt und Ernst Stiftung fondée en 2001 par Lola et Bengt Schwitters (respec-tivement la veuve du fils de Schwitters et son petit-fils). Il y a plus encore: les archives données par Ernst Schwitters en 1993, qui comportent entre autres un gigantesque fichier - des mètres d'armoires métalliques remplies de dossiers suspendus - auquel il travaillait depuis le début des années 1960. Ernst Schwitters avait mis au point un système très opérationnel de fiches dotées, sur le bord supérieur, d'un code coloré permettant de saisir en un coup d'œil les principales caractéristiques de l'œuvre, tandis que la fiche elle-même développe les informations techniques, l'historique de l'œuvre, les expositions et un commentaire. Les 2000 fiches (dont 1700 pourvues d'une photographie) héritées par le Sprengel Museum ont été vérifiées de façon critique et complétées avant d'être intégrées à la banque de données adoptée pour l'établissement du catalogue raisonné (le système DISKUS - Digitalen Informationssystem für Kunstund Sozialgeschichte). Leur nombre a quasiment doublé, puisque le troisième et dernier volume s'arrête au numéro 3664. Cela s'explique par la prise en compte des tableaux et dessins figuratifs, que le fils de l'artiste avait volontairement écartés, et par 
des recherches systématiques qui ont permis d'identifier quantité d'œuvres, dont certaines aujourd'hui détruites ou disparues.

4 La typographie et la mise en pages sobres et parfaitement claires font de ce catalogue raisonné un modèle d'élégance et d'efficacité visuelle: chaque page présente trois œuvres par le biais d'une vignette en noir et blanc et d'une colonne de texte. L'ensemble est rythmé par des cahiers couleurs où des œuvres importantes sont reproduites en pleine page. L'excellente qualité des reproductions mérite d'être soulignée. Nul besoin de lire les «Erläuterungen zum Catalogue raisonné» [Explications sur le catalogue raisonné] pour l'employer de façon optimale - mais quiconque s'y attèle sera convaincu par le sérieux de l'entreprise, par les choix méthodologiques et la science des auteurs.

5 Se distinguant par leur jaquette, dont la valeur de gris monte d'un ton à chaque fois, les volumes couvrent trois périodes distinctes, artistiquement et historiquement fondées. Ce parti pris chronologique est reconduit à l'intérieur de chacune d'entre elles, puis doublé d'un classement par techniques, thèmes et genres. Pour une même année, on trouve d'abord les tableaux, qui rassemblent successivement les assemblages, reliefs et tableaux à l'huile. Ces derniers sont répartis en tableaux abstraits et figuratifs, euxmêmes subdivisés en tableaux de genre, paysages, intérieurs, natures mortes et portraits. Ce classement instaure une hiérarchie qui est celle du Modernisme (les assemblages - «Tableaux Merz » - occupent la première place, les tableaux abstraits passent avant les tableaux figuratifs), mais uniquement à l'échelle de la production annuelle et non de l'œuvre tout entier, ce qui a le mérite de rendre compte de la permanence de la pratique figurative, et de sa proportion, souvent liée à la situation de l'artiste. Les tableaux sont suivis par les œuvres sur papier, subdivisées elles aussi en techniques dont la succession est fonction de leur degré d'innovation, des collages aux dessins, répartis en feuilles abstraites et figuratives. Viennent enfin les œuvres en trois dimensions avec notamment les sculptures et les Merzbauten (Constructions Merz), documentés par des photographies et des plans.

6 Pour chaque œuvre, tout a été passé au peigne fin : titre, date, catégorie, technique, présentation (passe-partout, cadre, socle), dimensions, annotations manuscrites, archives non publiées en rapport, provenance, littérature, expositions. À titre d'exemple, 1200 catalogues d'expositions, personnelles et collectives, servent de référence et la littérature se fonde sur un corpus de 2000 titres - qui prend en compte les travaux universitaires non publiés.

7 Dans la foulée de la sortie du troisième volume, Isabel Schulz et Karin Orchard ont organisé une exposition leur permettant de sortir du système clos du catalogue raisonné pour aborder Schwitters en contexte: 'Merzgebiete'. Kurt Schwitters und seine Freunde envisage les terres d'élection de Schwitters et met au jour tout son réseau amical et professionnel: "En dépit des nombreuses rétrospectives Schwitters organisées ces dernières années, déclarent les commissaires, la naissance et le développement de son œuvre ne furent encore jamais présentés en relation avec les tendances artistiques de son temps ${ }^{7}$. Malgré une mise en pages quelconque, les nombreuses reproductions du catalogue mettent bien en évidence les dialogues entre Schwitters et les expressionnistes, cubistes, dadaïstes, constructivistes et abstraits de tous pays, etc. Si effectivement ces confrontations n'avaient jamais fait l'objet d'une exposition à part entière, en revanche, elles ont été largement commentées et étudiées par les historiens de l'art. Dans leur ensemble, les articles du catalogue se font l'écho de 
l'état des connaissances en ce domaine; certains toutefois s'appuient sur des sources moins connues. Ainsi Karin Orchard enrichit-elle la question des liens entre Schwitters et le milieu de l'art par la divulgation de ses carnets d'adresses (organisés non par ordre alphabétique, mais par régions, les «Merzgebiete» qui donnent leur titre à l'exposition); Isabel Schulz reconstitue la collection de Schwitters, qui n'était pas une simple accumulation d'œuvres d'amis, mais qui avait été sciemment constituée pour « orienter sur le nouvel art » (selon les mots de Schwitters); Gwendolen Webster livre une étude très précise de la réception du Merzbau par ses contemporains; enfin GötzLothar Darsow produit un document totalement inédit, une photographie des années 1930 d'une maquette d'un «Palais (ou étable) pour cochons d'Inde avec quelques architecturaux mobiles » réalisée par Laszlo Moholy-Nagy et Schwitters. Cela mis à part, les textes n'apportent guère d'éléments nouveaux. Tout au moins auraient-ils pu alors renouveler l'approche; malheureusement, face à des sujets trop vastes (Kurt Schwitters « et les Pays-Bas », " entre dadaïsme et construc-tivisme » et «ses anciens et nouveaux amis à Londres »), les auteurs se contentent de décrire les événements selon une grille d'analyse qui se répète d'un article à l'autre - les sous-titres sont d'ailleurs symptomatiques de la superficialité et de l'uniformité des approches.

Absente ici, l'ambition théorique préside en revanche au catalogue publié par Hartwig Fischer à l'occasion de l'exposition schwitters_arp, voici deux ans au Kunstmuseum de Bâle. Sont mises en évidence des problématiques qui travaillent les œuvres des deux artistes: à titre d'exemples, le devenir abstrait des "choses" est interrogé par Gottfried Böhm ; la mystique, le hasard et l'improvisation sont étudiés par Götz-Lothar Darsow, la place de l'écriture et du mot est analysée par Bruno Haas. On saisit alors les points communs, sans que les différences soient passées sous silence, on se rend compte que parenté conceptuelle et parenté formelle ne sont pas obligatoirement liées ; la connaissance de chaque œuvre s'en trouve enrichie sans jamais tomber dans des rapprochements formels convenus ou des lieux communs. Plus osée, l'approche gagne en souffle - qui n'est toutefois pas totalement absent du catalogue de Hanovre, grâce à la traduction d'un extrait du Narré des îles Schwitters de Patrick Beurard-Valdoye à paraître prochainement aux éditions $\mathrm{Al}$ Dante, et dont plusieurs extraits ont d'ores et déjà été publiés dans des revues telles que L'Animal, Mouvement, L'étrangère.

Peut-être faut-il rappeler que P. Beurard-Valdoye fut un temps historien et critique d'art avant de se consacrer exclusivement à sa pratique poétique qui culmine dans Le cycle des exils, dont Le narré des îles Schwitters constitue le cinquième volume. C'est précisément la période des exils qu'il entreprend de restituer en s'appuyant sur la méthode qui est désormais la sienne, très rigoureuse et digne du plus méticuleux des historiens. Plusieurs années de recherche lui ont été nécessaires pour éplucher toute la littérature secondaire, mais aussi les archives (et pas seulement celles de Schwitters), pour lire tous les écrits de l'artiste et ses correspondances, pour rencontrer les spécialistes, mais aussi les derniers témoins ou leurs descendants, pour se rendre sur chaque lieu, en observer les moindres détails et en retenir les atmosphères, pour ausculter les œuvres, qui dans le cas de Schwitters renferment autant d'indices que d'énigmes - soit dit en passant, en tous ces domaines, P. Beurard-Valdoye est souvent allé plus loin que les chercheurs patentés, il a d'ailleurs fait plusieurs découvertes et précisé bien des points. Tel est le matériau à partir duquel il a construit son œuvre : une prose poétique qui court sur plus de trois cents pages et qui se nourrit des acquis modernes, un langage " dépaysé " par le procédé du collage (ainsi surgissent tels quels des documents d'archive ou des mots piochés dans les œuvres et dans les écrits de 
l'artiste), par l'intercalation de mots étrangers (allemand, norvégien, anglais), par un travail sur la ponctuation, des jeux typographiques, etc. Proche d'une certaine poésie phonique, Le narré des îles Schwitters se lie l'oreille tendue, toutefois ces jeux formels ne sont jamais une fin en soi : la poésie de P. Beurard-Valdoye est porteuse de sens et d'une certaine manière de l'expression de la condition humaine.

Ce travail pose en outre la question, sensible à tout historien de l'art, de la restitution d'une œuvre d'art partiellement ou totalement détruite. Un passage est ainsi consacré au deuxième Merzbau que Schwitters avait entrepris en 1930, à Lysaker, et qui fut malencontreusement incendié dans les années 1950. En l'absence de toute photographie, Beurard-Valdoye se fonde cette fois sur peu de chose: un texte de Schwitters, un plan schématique, un vague témoignage, des repérages sur place, l'hypothèse d'invariants communs aux divers Merzbauten, et un "inventaire de sensations » constitué notamment lors de la visite de la ruine du Merzbau 3 sur l'île de Hjertöya. Alors qu'aucun historien de l'art ne s'était aventuré à écrire sur cette pièce, ni même n'avait risqué une projection, à l'instar des archéologues ou restaurateurs confrontés à quelques fragments, Patrick Beurard-Valdoye, après mûre réflexion, s'autorise "à réinventer 'quelque chose' dans le champ des arts poétiques ", décidant à la fois « de franchir l'interdit, [d']outrepasser les usages et les méthodes scientifiques » et de mettre à l'épreuve sa propre méthode: "Jusqu'à quel point, se demande-t-il, pouvais-je 'inventer', en absence d'informations, de preuves (de traces) ?» - car pour lui, « le poète, contrairement à ce que prétend la tradition, ne ment pas $»^{8}$. Or, bien qu'inventée, la description du Merzbau 2 ne ment pas, pour la simple et bonne raison que l'auteur, ici comme partout ailleurs dans son œuvre, ne triche pas avec la vérité des sensations ressenties au contact d'une œuvre, d'un artiste, face à l'histoire des hommes et des pays où ils vivent.

11 P. Beurard-Valdoye nous ferait presque oublier que la France continue à faire la fine bouche - dans le domaine éditorial, rien de neuf depuis le catalogue publié par Serge Lemoine au moment de la rétrospective organisée au MNAM en 1994 et le travail de traduction des écrits de Schwitters par Marc Dachy. Il y parvient d'autant mieux qu'il interroge la question de l'écriture de l'histoire de l'art et adopte une position originale, celle-là même que préconisait Schwitters: "Moi-même j'ai une préférence pour la critique qui est elle-même œuvre d'art, c'est-à-dire une œuvre analogue à l'œuvre critiquée, mais avec les moyens du langage [...]. Une bonne critique doit pouvoir supporter la comparaison avec l'œuvre d'art critiquée $»^{9}$.

\section{NOTES}

1. Karin Orchard, Isabel Schulz, Catalogue raisonné. Kurt Schwitters. Band 1 1905-1922 ; Band 2. 1923-1936 ; Band 3. 1937-1948, Ostfildern-Ruit, Hatje Cantz Verlag, 2000, 2003, 2006.

2. Karin Orchard, Isabel Schulz (sous la dir.), Merzgebiete. Kurt Schwitters und seine Freunde, (cat. expo., Hanovre, Sprengel Museum, 2006), Hanovre, 2006.

3. Hartwig Fischer éd., Schwitters_arp, (cat. expo., Bâle, Kunstmuseum), Bâle/Ostfildern-Ruit, Hatje Cantz Verlag, 2004. 
4. Patrick Beurard-Valdoye, Le Narré des îles Schwitters, Paris, Al Dante, 2006.

5. Lettre de Schwitters à Herwarth Walden, 1.12.1920, reproduite dans Ernst Nündel éd., Kurt Schwitters. Wir spielen, bis uns der Todf abholt. Briefe aus fünf Jahrzehnten, Francfort-sur-le-Main, 1986, p. 42.

6. Voir Werner Schmalenbach, «Le retour de Hanovre ", dans Serge Lemoine éd., Kurt Schwitters, (cat. expo., Paris, Musée national d'art moderne, 1994-1995), Paris, 1994, p. 366-367.

7. Karin Orchard, Isabel Schulz, «Einleitung ", dans Merzgebiete. Kurt Schwitters und seine Freunde, (cat. expo., Hanovre, Sprengel Museum, 2006), Hanovre, 2006, p. 14-15.

8. Patrick Beurard-Valdoye, «Ekphrasis ", dans Les interdits de l'image, Chevillon, 2006, p. 64.

9. Kurt Schwitters, «Über den Wert der Kritik (Nachtrag). Meine Ansicht über den Wert der Kunstkritik (Für den Ararat) », dans Der Ararat, II, n5, mai 1921, cité d'après Friedhelm Lach éd., Kurt Schwitters. Das literarische Werk. Manifeste und kritische Prosa, Cologne, 1981, p. 88.

INDEX

Keywords : monograph, perpetuation, restitution, biography, historiography Mots-clés : monographie, pérennisation, restitution, biographie, historiographie Index chronologique : 1900

\section{AUTEUR}

ISABELLE EWIG

Université Paris IV-Sorbonne 